\title{
BETHE ANSATZ FOR ARRANGEMENTS OF HYPERPLANES AND THE GAUDIN MODEL
}

\author{
ALEXANDER VARCHENKO *,1

\begin{abstract}
We show that the Shapovalov norm of a Bethe vector in the Gaudin model is equal to the Hessian of the logarithm of the corresponding master function at the corresponding isolated critical point. We show that different Bethe vectors are orthogonal. These facts are corollaries of a general Bethe ansatz type construction, suggested in this paper and associated with an arbitrary arrangement of hyperplanes.
\end{abstract} \\ * Department of Mathematics, University of North Carolina at Chapel Hill, \\ Chapel Hill, NC 27599-3250, USA
}

July, 2004

\section{INTRODUCTION}

The Bethe ansatz is a large collection of methods in the theory of quantum integrable models to calculate the spectrum and eigenvectors for a certain commutative sub-algebra of observables for an integrable model. Elements of the sub-algebra are called Hamiltonians, or integrals of motion, or conservation laws of the model. The bibliography on the Bethe ansatz method is enormous, see for example [BIK, [Fa, [FT].

In the theory of the Bethe ansatz one assigns the Bethe ansatz equations to an integrable model. Then a solution of the Bethe ansatz equations gives an eigenvector of commuting Hamiltonians of the model. The general conjecture is that the constructed vectors form a basis in the space of states of the model.

The simplest and interesting example is the Gaudin model associated with a complex simple Lie algebra $\mathfrak{g}$, see $\mathrm{B}, \mathrm{BF}, \mathrm{F}, \mathrm{FFR}, \mathrm{G}, \mathrm{MV2}, \mathrm{MV3}, \mathrm{MV}$, $\mathrm{RV}, \mathrm{ScV}, \mathrm{V2}$, V3]. One considers highest weight $\mathfrak{g}$-modules $V_{\Lambda_{1}}, \ldots, V_{\Lambda_{n}}$ and their tensor product $V_{\boldsymbol{\Lambda}}$. One fixes a point $z=\left(z_{1}, \ldots, z_{n}\right) \in \mathbb{C}^{n}$ with distinct coordinates and defines linear operators $K_{1}(z), \ldots, K_{n}(z)$ on $V_{\boldsymbol{\Lambda}}$ by the formula

$$
K_{i}(z)=\sum_{j \neq i} \frac{\Omega^{(i, j)}}{z_{i}-z_{j}}, \quad i=1, \ldots, n .
$$

Here $\Omega^{(i, j)}$ is the Casimir operator acting in the $i$-th and $j$-th factors of the tensor product. The operators are called the Gaudin Hamiltonians of the Gaudin model associated with $V_{\boldsymbol{\Lambda}}$. The Hamiltonians commute.

\footnotetext{
${ }^{1}$ Supported in part by NSF grant DMS-0244579.
} 
The common eigenvectors of the Gaudin Hamiltonians are constructed by the Bethe ansatz method. Namely, one assigns to the model a scalar function $\Phi(t, z)$ of new auxiliary variables $t$ and a $V_{\boldsymbol{\Lambda}}$-valued function $\omega(t, z)$ such that $\omega\left(t^{0}, z\right)$ is an eigenvector of the Hamiltonians if $t^{0}$ is a critical point of $\Phi$. The functions $\Phi$ and $\omega$ were introduced in [SV] to construct hypergeometric solutions of the $\mathrm{KZ}$ equations. The function $\Phi$ is called the master function and the function $\omega$ is called the canonical weight function.

The first question is if the Bethe eigenvector $\omega\left(t^{0}, z\right)$ is non-zero. In this paper we show that the Bethe vector is non-zero if $t^{0}$ is a non-degenerate critical point of the master function $\Phi$. To show that we prove (in part (i) of Theorem 5.2) the following identity:

$$
S\left(\omega\left(t^{0}, z\right), \omega\left(t^{0}, z\right)\right)=\operatorname{Hess}_{t} \ln \Phi\left(t^{0}, z\right) .
$$

Here $S$ is the tensor product Shapovalov form on the tensor product $V_{\boldsymbol{\Lambda}}$ and the right hand side of the formula is the Hessian at $t^{0}$ of the function $\ln \Phi$.

This formula for the Gaudin model, associated with $\mathfrak{g}=s l_{r+1}$, was proved in V2], if $r=1$, and for arbitrary $r$ in [MV4], see also [Ko, R, RV], TV], MV1].

We also show (in part (ii) of Theorem 5.2) that different Bethe vectors are orthogonal with respect to the tensor product Shapovalov form.

These two statements allow us to reduce the Bethe ansatz conjecture to a question about the number of non-degenerate critical points of the master function, see part (iii) of Theorem 5.2

The formulated statements on the Bethe vectors are corollaries of a general construction, suggested in this paper and related to an arbitrary arrangement of hyperplanes. Namely, let $\mathcal{C}$ be an arrangement of affine hyperplanes in $\mathbb{C}^{k}$ having a vertex. One defines the Orlik-Solomon algebra $\mathcal{A}(\mathcal{C})=\oplus_{p} \mathcal{A}^{p}(\mathcal{C})$ and the flag space $\mathcal{F}(\mathcal{C})=\oplus_{p} \mathcal{F}^{p}(\mathcal{C})$ in the standard way, $[\mathrm{SV}$.

The spaces $\mathcal{A}^{p}(\mathcal{C})$ and $\mathcal{F}^{p}(\mathcal{C})$ are dual. We are interested in the top degree spaces $\mathcal{A}^{k}(\mathcal{C})$ and $\mathcal{F}^{k}(\mathcal{C})$.

Assume that a complex number $a(H)$ is assigned to every hyperplane $H$ of $\mathcal{C}$. Then one can define a symmetric bilinear form $S^{(a)}: \mathcal{F}^{k}(\mathcal{C}) \otimes \mathcal{F}^{k}(\mathcal{C}) \rightarrow \mathbb{C}$ called the Shapovalov form of $\mathcal{C}, \underline{\mathrm{SV}}]$. One also defines the master function of $\mathcal{C}, \Phi=\prod_{H \in \mathcal{C}} f_{H}^{a(H)}$, where $f_{H}=0$ is the defining equation of the hyperplane $H$.

Let $t_{1}, \ldots, t_{k}$ be coordinates in $\mathbb{C}^{k}$. Remind that the space $\mathcal{A}^{k}(\mathcal{C})$ is the space of rational differential $k$-forms on $\mathbb{C}^{k}$ which can be written as exterior polynomials in differential 1-forms $d f_{H} / f_{H}, H \in \mathcal{C}$. Hence each $\eta \in \mathcal{A}^{k}(\mathcal{C})$ can be written as $u d t_{1} \wedge$ $\cdots \wedge d t_{k}$ where $u$ is a rational function.

Define the rational map $v: \mathbb{C}^{k} \rightarrow \mathcal{F}^{k}(\mathcal{C})$, regular on the complement to the union of hyperplanes, as follows. Let $\epsilon \in \mathcal{A}^{k}(\mathcal{C}) \otimes \mathcal{F}^{k}(\mathcal{C})$ be the canonical element, $\epsilon=$ $\sum_{m} x_{m}^{*} \otimes x_{m}$ where $\left\{x_{m}\right\}$ is a basis in $\mathcal{F}^{k}(\mathcal{C})$ and $\left\{x_{m}^{*}\right\}$ is the dual basis in $\mathcal{A}^{k}(\mathcal{C})$. If $x_{m}^{*}=u_{m} d t_{1} \wedge \cdots \wedge d t_{k}$, then $v(t)=\sum_{m} u_{m}(t) x_{m}$. 
In part (ii) of Theorem 3.1 we show that

$$
S^{(a)}(v(t), v(t))=(-1)^{k} \operatorname{det}_{1 \leq i, j \leq k}\left(\frac{\partial^{2}}{\partial t_{i} \partial t_{j}} \ln \Phi\right)(t) .
$$

In part (iii) of Theorem 3.1 we show that if $t^{1}, t^{2}$ are different isolated critical points of $\Phi$, then the special vectors $v\left(t^{1}\right), v\left(t^{2}\right)$ are orthogonal, $S^{(a)}\left(v\left(t^{1}\right), v\left(t^{2}\right)\right)=0$.

Theorem 3.1 is the main result of the paper. To obtain the results concerning the Bethe ansatz for the Gaudin model we apply Theorem 3.1 to discriminantal arrangements following methods of [SV].

In this paper we considered the Bethe ansatz associated with a simple Lie algebra. In the same way one may consider the case of an arbitrary Kac-Moody algebra. The statements and proofs remain the same.

The paper is organized as follows. Section 2 contains basic facts about the OrlikSolomon algebra and flag spaces of an arrangement. Section 3 contains the construction of special singular vectors in the top flag space and the statement of Theorem 3.1. In Section 4 we prove Theorem 3.1. Section 5 contains applications of Theorem 3.1] to the Bethe ansatz associated with the Gaudin model.

The idea of this paper was formulated long time ago in $\nabla 2$, where it was mentioned that an analog of the Bethe ansatz construction must exist for an arbitrary arrangement of hyperplanes.

The author thanks IHES for warm hospitality.

\section{Arrangements, SV, V1}

2.1. Arrangement. Let $\mathcal{C}=\left\{H_{j}\right\}, j \in J(\mathcal{C})$, be an arrangement of affine hyperplanes in the complex affine space $\mathbb{C}^{k}$. Denote by $U$ the complement to the union of all hyperplanes,

$$
U=\mathbb{C}^{k}-\cup_{j \in J(\mathcal{C})} H_{j}
$$

Hyperplanes $H_{j}$ define in $\mathbb{C}^{k}$ the structure of a stratified space. A closed stratum $X_{\alpha} \subset$ $\mathbb{C}^{k}$ is the intersection of some hyperplanes $H_{j}, j \in J_{\alpha} \subset J(\mathcal{C})$. For a stratum $X_{\alpha}$ we denote $l\left(X_{\alpha}\right)=\operatorname{codim}_{\mathbb{C}^{k}} X_{\alpha}$. In this paper we will always assume that $\mathcal{C}$ has a vertex, a stratum of dimension 0 .

2.2. Orlik-Solomon algebra. Define complex vector spaces $\mathcal{A}^{p}(\mathcal{C}), p=0, \ldots, k$. For $p=0$ set $\mathcal{A}^{p}(\mathcal{C})=\mathbb{C}$. For $p \geq 1, \mathcal{A}^{p}(\mathcal{C})$ is generated by symbols $\left(H_{i_{1}}, \ldots, H_{i_{p}}\right)$ with $H_{i_{j}} \in \mathcal{C}$, such that

(i) $\left(H_{i_{1}}, \ldots, H_{i_{p}}\right)=0$ if $H_{i_{1}}, \ldots, H_{i_{p}}$ are not in general position, that is if the intersection $H_{i_{1}} \cap \ldots \cap H_{i_{p}}$ is empty or its codimension is less than $p$;

(ii) $\left(H_{i_{\sigma(1)}}, \ldots, H_{i_{\sigma(p)}}\right)=(-1)^{|\sigma|}\left(H_{i_{1}}, \ldots, H_{i_{p}}\right)$ for any permutation $\sigma \in S_{p}$; 
(iii) $\sum_{j=1}^{p+1}(-1)^{j}\left(H_{i_{1}}, \ldots, \widehat{H}_{i_{j}}, \ldots, H_{i_{p+1}}\right)=0$ for any $(p+1)$-tuple $H_{i_{1}}, \ldots, H_{i_{p+1}}$ of hyperplanes in $\mathcal{C}$ which are not in general position and such that $H_{i_{1}} \cap \ldots \cap H_{i_{p+1}} \neq \emptyset$.

The direct sum $\mathcal{A}(\mathcal{C})=\oplus_{p=1}^{N} \mathcal{A}^{p}(\mathcal{C})$ is a graded skew commutative algebra with respect to the multiplication

$$
\left(H_{i_{1}}, \ldots, H_{i_{p}}\right) \cdot\left(H_{i_{p+1}}, \ldots, H_{i_{p+q}}\right)=\left(H_{i_{1}}, \ldots, H_{i_{p}}, H_{i_{p+1}}, \ldots, H_{i_{p+q}}\right) .
$$

The algebra is called the Orlik-Solomon algebra of the arrangement $\mathcal{C}$.

Let $a: \mathcal{C} \rightarrow \mathbb{C}$ be a map which assigns to each hyperplane $H$ a complex number $a(H)$ called the exponent of $H$. Set

$$
\omega(a)=\sum_{H \in \mathcal{C}} a(H) H \quad \in \mathcal{A}^{1}(\mathcal{C}) .
$$

The multiplication by $\omega(a)$ defines a differential

$$
d_{\mathcal{A}}^{(a)}: \mathcal{A}^{p}(\mathcal{C}) \rightarrow \mathcal{A}^{p+1}(\mathcal{C}), \quad x \mapsto \omega(a) \cdot x
$$

in the vector space of the Orlik-Solomon algebra.

It is known that for generic exponents $a, H^{p}\left(\mathcal{A}^{\bullet}(\mathcal{C}), d_{\mathcal{A}}^{(a)}\right)=0$ if $p<k$ and $\operatorname{dim} H^{p}\left(\mathcal{A}^{\bullet}, d_{\mathcal{A}}^{(a)}\right)=|\chi(U)|$, where $\chi(U)$ is the Euler characteristics of $U$, see [A], STV].

2.3. Space of Flags. For a stratum $X_{\alpha}, l\left(X_{\alpha}\right)=p$, a flag starting at $X_{\alpha}$ is a sequence

$$
X_{\alpha_{0}} \supset X_{\alpha_{1}} \supset \cdots \supset X_{\alpha_{p}}=X_{\alpha}
$$

of strata such that $l\left(X_{\alpha_{j}}\right)=j$ for $j=0, \ldots, p$.

For a stratum $X_{\alpha}$, we define $\overline{\mathcal{F}}_{X_{\alpha}}$ as the complex vector space with basis vectors

$$
\bar{F}_{X_{\alpha_{0}}, \ldots, X_{\alpha_{p}}=X_{\alpha}}
$$

labeled by the elements of the set of all flags starting at $X_{\alpha}$.

Define $\mathcal{F}_{X_{\alpha}}$ as the quotient of $\overline{\mathcal{F}}_{X_{\alpha}}$ over the subspace generated by the vectors

$$
\sum_{X_{\beta}, X_{\alpha_{j-1}} \supset X_{\beta} \supset X_{\alpha_{j+1}}} \bar{F}_{X_{\alpha_{0}}, \ldots, X_{\alpha_{j-1}}, X_{\beta}, X_{\alpha_{j+1}}, \ldots, X_{\alpha_{p}}=X_{\alpha}} .
$$

Such a vector is determined by $j \in\{1, \ldots, p-1\}$ and an incomplete flag $X_{\alpha_{0}} \supset \ldots \supset$ $X_{\alpha_{j-1}} \supset X_{\alpha_{j+1}} \supset \ldots \supset X_{\alpha_{p}}=X_{\alpha}$ with $l\left(X_{\alpha_{i}}\right)=i$.

Denote by $F_{X_{\alpha_{0}}, \ldots, X_{\alpha_{p}}}$ the image in $\mathcal{F}_{\alpha}$ of the basis vector $\bar{F}_{X_{\alpha_{0}}, \ldots, X_{\alpha_{p}}}$. Set

$$
\mathcal{F}^{p}(\mathcal{C})=\oplus_{X_{\alpha}, l\left(X_{\alpha}\right)=p} \mathcal{F}_{X_{\alpha}}, \quad \mathcal{F}(\mathcal{C})=\oplus_{p=0}^{k} \mathcal{F}^{p}(\mathcal{C}) .
$$

The direct sum

$$
\mathcal{F}(\mathcal{C})=\oplus_{p=0}^{k} \mathcal{F}^{p}(\mathcal{C})
$$

is a complex with respect to the differential

$$
d_{\mathcal{F}}: \mathcal{F}^{p}(\mathcal{C}) \rightarrow \mathcal{F}^{p+1}(\mathcal{C}), \quad F_{X_{\alpha_{0}}, \ldots, X_{\alpha_{p}}} \mapsto \sum_{X_{\alpha_{p+1}}, X_{\alpha_{p}} \supset X_{\alpha_{p+1}}} F_{X_{\alpha_{0}}, \ldots, X_{\alpha_{p}}, X_{\alpha_{p+1}}}
$$


2.4. Duality. The vector spaces $\mathcal{A}^{p}(\mathcal{C})$ and $\mathcal{F}^{p}(\mathcal{C})$ are dual. The pairing $\mathcal{A}^{p}(\mathcal{C}) \otimes$ $\mathcal{F}^{p}(\mathcal{C}) \rightarrow \mathbb{C}$ is defined as follows. For $H_{i_{1}}, \ldots, H_{i_{p}}$ in general position, set $F\left(H_{i_{1}}, \ldots, H_{i_{p}}\right)=$ $F_{X_{\alpha_{0}}, \ldots, X_{\alpha_{p}}}$ where

$$
X_{\alpha_{0}}=\mathbb{C}^{k}, \quad X_{\alpha_{1}}=H_{i_{1}}, \quad \ldots, \quad X_{\alpha_{p}}=H_{i_{1}} \cap \cdots \cap H_{i_{p}} .
$$

Then set $\left\langle\left(H_{i_{1}}, \ldots, H_{i_{p}}\right), F\right\rangle=(-1)^{|\sigma|}$, if $F=F\left(H_{i_{\sigma(1)}}, \ldots, H_{i_{\sigma(p)}}\right)$ for some $\sigma \in S_{p}$, and $\left\langle\left(H_{i_{1}}, \ldots, H_{i_{p}}\right), F\right\rangle=0$ otherwise.

Define the map $\delta_{\mathcal{F}}^{(a)}: \mathcal{F}^{p}(\mathcal{C}) \rightarrow \mathcal{F}^{p-1}(\mathcal{C})$ to be the map adjoint to $d_{\mathcal{A}}^{(a)}: \mathcal{A}^{p-1}(\mathcal{C}) \rightarrow$ $\mathcal{A}^{p}(\mathcal{C})$.

An element $v \in \mathcal{F}^{k}(\mathcal{C})$ will be called singular if $\delta_{\mathcal{F}}^{(a)} v=0$. Denote by $\operatorname{Sing} \mathcal{F}^{k}(\mathcal{C}) \subset$ $\mathcal{F}^{k}(\mathcal{C})$ the subspace of singular vectors.

For generic exponents $a$ the dimension of $\operatorname{Sing} \mathcal{F}^{k}(\mathcal{C})$ is equal to $|\chi(U)|$.

2.5. The Shapovalov map and form. The collection of exponents a determines the Shapovalov map

$$
\mathcal{S}^{(a)}: \mathcal{F}(\mathcal{C}) \rightarrow \mathcal{A}(\mathcal{C}), \quad F_{X_{\alpha_{0}}, \ldots, X_{\alpha_{p}}} \mapsto \sum a\left(H_{i_{1}}\right) \cdots a\left(H_{i_{p}}\right)\left(H_{i_{1}}, \ldots, H_{i_{p}}\right),
$$

where the sum is taken over all $p$-tuples $\left(H_{i_{1}}, \ldots, H_{i_{p}}\right)$ such that

$$
H_{i_{1}} \supset X_{a_{1}}, \ldots, H_{i_{p}} \supset X_{\alpha_{p}} .
$$

According to $\underline{S V}$, the map $\mathcal{S}^{(a)}$ is a morphism of the complex $\left(\mathcal{F}^{\bullet}(\mathcal{C}), d_{\mathcal{F}}\right)$ to the complex $\left(\mathcal{A}^{\bullet}(\mathcal{C}), d_{\mathcal{A}}^{(a)}\right)$. The image $\left(\mathcal{S}^{(a)}\left(\mathcal{F}^{\bullet}(\mathcal{C})\right), d_{\mathcal{A}}^{(a)}\right)$ is called the complex of flag forms of $\mathcal{C}$.

Identifying $\mathcal{A}(\mathcal{C})$ with $\mathcal{F}(\mathcal{C})^{*}$, we may consider the map $\mathcal{S}^{(a)}$ as a bilinear form on the vector space $\mathcal{F}(\mathcal{C})$. This bilinear form, denoted by $S^{(a)}$, is symmetric and is called the Shapovalov form.

If $F_{1}, F_{2} \in \mathcal{F}^{p}(\mathcal{C})$, then

$$
S^{(a)}\left(F_{1}, F_{2}\right)=\sum_{\left\{i_{1}, \ldots, i_{p}\right\} \subset J(\mathcal{C})} a\left(H_{i_{1}}\right) \cdots a\left(H_{i_{p}}\right)\left\langle\left(H_{i_{1}}, \ldots, H_{i_{p}}\right), F_{1}\right\rangle\left\langle\left(H_{i_{1}}, \ldots, H_{i_{p}}\right), F_{2}\right\rangle,
$$

where the sum is over all unordered $p$-element sets.

\section{Master Function, Special Vectors}

3.1. Master function. Let $\mathcal{C}=\left\{H_{j}\right\}, j \in J(\mathcal{C})$, be an arrangement of affine hyperplanes in $\mathbb{C}^{k}$. For $j \in J(\mathcal{C})$, fix a defining equation for $H_{j}, f_{j}=0$. Let $a: \mathcal{C} \rightarrow \mathbb{C}$ be a set of exponents. Then the function

$$
\Phi=\prod_{j \in J(\mathcal{C})} f_{j}^{a\left(H_{j}\right)}
$$

is called the master function. The master function is a multi-valued function defined on $U$.

A point $t \in U$ is called a critical point of $\Phi$ if $\left.d \Phi\right|_{t}=0$. 
Fix affine coordinates $t_{i}, i=1, \ldots, k$, on $\mathbb{C}^{k}$. For $j \in J(\mathcal{C})$, we have

$$
f_{j}\left(t_{1}, \ldots, t_{k}\right)=b_{j}^{0}+b_{j}^{1} t_{1}+\cdots+b_{j}^{k} t_{k}, \quad b_{j}^{i} \in \mathbb{C} .
$$

A point $t \in U$ is a critical point of $\Phi$ if and only if

$$
\sum_{j \in J(\mathcal{C})} \frac{\partial f_{j}}{\partial t_{i}} \frac{a\left(H_{j}\right)}{f_{j}}=0, \quad i=1, \ldots k
$$

at $t$.

It is known that for generic exponents $a$ all critical points of $\Phi$ are non-degenerate and their number is equal to $|\chi(U)|$, see [V2, OT, Si].

3.2. Realization of the Orlik-Solomon algebra. For $j \in J(\mathcal{C})$, consider the logarithmic differential form $\omega_{j}=d f_{j} / f_{j}$ on $\mathbb{C}^{k}$. Let $\overline{\mathcal{A}}(\mathcal{C})$ be the graded $\mathbb{C}$-algebra with unit element generated by all $\omega_{j}$ 's. The map $\mathcal{A}(\mathcal{C}) \rightarrow \overline{\mathcal{A}}(\mathcal{C}), H_{j} \mapsto \omega_{j}$, is an isomorphism. We shall identify $\mathcal{A}(\mathcal{C})$ and $\overline{\mathcal{A}}(\mathcal{C})$.

3.3. Special vectors in $\mathcal{F}^{k}(\mathcal{C})$. A top degree form $\eta \in \mathcal{A}^{k}(\mathcal{C})$ can be written as

$$
\eta=u d t_{1} \wedge \cdots \wedge d t_{k}
$$

where $u$ is a rational function regular on $U$.

Define the rational map $v: \mathbb{C}^{k} \rightarrow \mathcal{F}^{k}(\mathcal{C})$, regular on $\mathrm{U}$, as follows. For $t \in U$, set $v(t)$ to be the element of $\mathcal{F}^{k}(\mathcal{C})$ such that

$$
\langle\eta, v(t)\rangle=u(t) \quad \text { for any } \eta \in \mathcal{A}^{k}(\mathcal{C}) \text {. }
$$

Let $\epsilon \in \mathcal{A}^{k}(\mathcal{C}) \otimes \mathcal{F}^{k}(\mathcal{C})$ be the canonical element, $\epsilon=\sum_{m} x_{m}^{*} \otimes x_{m}$ where $\left\{x_{m}\right\}$ is a basis in $\mathcal{F}^{k}(\mathcal{C})$ and $\left\{x_{m}^{*}\right\}$ is the dual basis in $\mathcal{A}^{k}(\mathcal{C})$. If $x_{m}^{*}=u_{m} d t_{1} \wedge \cdots \wedge d t_{k}$, then $v(t)=\sum_{m} u_{m}(t) x_{m}$.

The map $v$ will be called the specialization map, its value $v(t)$ will be called the special vector associated with $t \in U$.

Define the rational function $\operatorname{Hess}^{(\mathrm{a})}: \mathbb{C}^{k} \rightarrow \mathbb{C}$, regular on $U$, by the formula

$$
\operatorname{Hess}^{(\mathrm{a})}(t)=\operatorname{det}_{1 \leq i, j \leq k}\left(\frac{\partial^{2}}{\partial t_{i} \partial t_{j}} \ln \Phi\right)(t) .
$$

\section{Theorem 3.1.}

(i) A point $t \in U$ is a critical point of $\Phi$, if and only if the special vector $v(t)$ is a singular vector.

(ii) If $t \in U$, then

$$
S^{(a)}(v(t), v(t))=(-1)^{k} \operatorname{Hess}^{(\mathrm{a})}(t) .
$$

(ii) If $t^{1}, t^{2} \in U$ are different isolated critical points of $\Phi$, then the special singular vectors $v\left(t^{1}\right), v\left(t^{2}\right)$ are orthogonal,

$$
S^{(a)}\left(v\left(t^{1}\right), v\left(t^{2}\right)\right)=0 .
$$

The theorem is proved in Section 4 . 
3.4. Shapovalov images of special vectors. . The composition of the specialization and Shapovalov maps define a rational map $\mathcal{S}^{(a)} v: \mathbb{C}^{k} \rightarrow \mathcal{A}^{k}(\mathcal{C})$ regular on $U$.

Lemma 3.1. For $t^{1}, t^{2} \in U$, we have

$$
\left.\mathcal{S}^{(a)} v\left(t^{1}\right)\right|_{t^{2}}=S^{(a)}\left(v\left(t^{1}\right), v\left(t^{2}\right)\right) d t_{1} \wedge \cdots \wedge d t_{k} .
$$

Proof. Let $t^{1}, t^{2} \in U$ and $\mathcal{S}^{(a)} v\left(t^{1}\right)=u d t_{1} \wedge \cdots \wedge d t_{k}$. Then $S^{(a)}\left(v\left(t^{1}\right), v\left(t^{2}\right)\right)=$ $\left\langle\mathcal{S}^{(a)} v\left(t^{1}\right), v\left(t^{2}\right)\right\rangle=u\left(t^{2}\right)$.

The following corollary gives an estimate from above on the number of non-degenerate critical points of $\Phi$ by the dimension of the vector space

$$
\mathcal{H}^{k}=\mathcal{S}^{(a)}\left(\mathcal{F}^{k}(\mathcal{C})\right) / d^{(a)} \mathcal{S}^{(a)}\left(\mathcal{F}^{k-1}(\mathcal{C})\right) .
$$

Corollary 3.2. Let $\mathcal{C}$ be an arrangement of affine hyperplanes in $\mathbb{C}^{k}$. Let $\mathrm{d}$ be a natural number. Let $C$ be a set of d non-degenerate critical points of $\Phi$. Then the natural projection of vectors $\left\{\mathcal{S}^{(a)} v(t)\right\}_{t \in C}$ span a d-dimensional subspace in $\mathcal{H}^{k}$.

In particular, if $\Phi$ has $d$ non-degenerate critical points, where $\mathrm{d}$ is the dimension of $\mathcal{H}^{k}$, then the natural projections to $\mathcal{H}^{k}$ of the vectors $\mathcal{S}^{(a)} v(t)$, associated with those points, form a basis in $\mathcal{H}^{k}$.

3.5. Basis of special singular vectors. The following corollary gives an estimate from above on the number of non-degenerate critical points of $\Phi$ by the dimension of the kernel of the linear operator $\delta_{\mathcal{F}}^{(a)}: \mathcal{F}^{k}(\mathcal{C}) \rightarrow \mathcal{F}^{k-1}(\mathcal{C})$.

Corollary 3.3. Let $\mathcal{C}$ be an arrangement of affine hyperplanes in $\mathbb{C}^{k}$. Let $\mathrm{d}$ be a natural number. Let $C$ be a set of d non-degenerate critical points of $\Phi$. Then the special singular vectors $\{v(t)\}_{t \in C}$ span a d-dimensional subspace in $\operatorname{Sing} \mathcal{F}^{k}(\mathcal{C})$.

In particular, if $\Phi$ has $\mathrm{d}$ non-degenerate critical points, where $\mathrm{d}$ is the dimension of Sing $\mathcal{F}^{k}(\mathcal{C})$. Then the special singular vectors, associated to those points, form a basis in $\operatorname{Sing} \mathcal{F}^{k}(\mathcal{C})$.

Corollary 3.4. If the exponents a are generic, then the set $\{v(t)\}_{t \in C}$ is a basis in Sing $\mathcal{F}^{k}(\mathcal{C})$.

3.6. Arrangements with symmetries. Assume that a finite group $G$ acts on $\mathbb{C}^{k}$ by affine linear transformations so that the arrangement $\mathcal{C}$ is preserved. Assume that exponents $a$ are preserved by this action, $a(g(H))=a(H)$ for $g \in G, H \in \mathcal{C}$.

The group $G$ naturally acts on $\mathcal{F}^{p}(\mathcal{C})$ for any $p$. The action on $\mathcal{F}^{k}(\mathcal{C})$ will be denoted by $R$. The action commutes with the differential $\delta_{\mathcal{F}}^{(a)}$. The subspace $\operatorname{Sing} \mathcal{F}^{k}(\mathcal{C}) \subset \mathcal{F}^{k}(\mathcal{C})$ is $G$-invariant. The Shapovalov form $S^{(a)}: \mathcal{F}^{k}(\mathcal{C}) \otimes \mathcal{F}^{k}(\mathcal{C}) \rightarrow \mathbb{C}$ is $G$-invariant.

Let $\Omega^{k}$ be the one dimensional complex vector space of differential $k$-forms on $\mathbb{C}^{k}$ invariant with respect to all affine translations. The action of $G$ on $\mathbb{C}^{k}$ determines a representation $\rho: G \rightarrow \mathbb{C}^{*}, g \mapsto \rho_{g}$, defined by the condition

$$
\rho_{g} g^{*}(\eta)=\eta, \quad \eta \in \Omega^{k}
$$


Fix affine coordinates $t_{i}, i=1, \ldots, k$, on $\mathbb{C}^{k}$. Let $v: \mathbb{C}^{k} \rightarrow \mathcal{F}^{k}(\mathcal{C})$ be the specialization map. We have

$$
v(g(t))=\rho_{g} R_{g}(v(t)), \quad t \in U, g \in G .
$$

The critical set $C \subset U$ of the master function $\Phi$ is $G$-invariant and

$$
\operatorname{Hess}^{(\mathrm{a})}(g(t))=\left(\rho_{g}\right)^{2} \operatorname{Hess}^{(\mathrm{a})}(t) .
$$

Corollary 3.5. Let $t \in U$ be a non-degenerate critical point of $\Phi$ and $\mathcal{O}$ its $G$-orbit. Let $W$ be the span in $\operatorname{Sing} \mathcal{F}^{k}(\mathcal{C})$ of the vectors $\left.\left\{v\left(t^{\prime}\right)\right\}\right|_{t^{\prime} \in \mathcal{O}}$. Then $W$ is $G$-invariant and $\operatorname{dim} W=|\mathcal{O}|$.

Let $\rho^{1}, \ldots, \rho^{N}$ be all distinct irreducible representations of $G, d_{1} \ldots, d_{N}$ the corresponding dimensions, $\chi_{1}, \ldots, \chi_{N}$ the corresponding characters, $\mathcal{F}^{k}(\mathcal{C})=W_{1} \oplus \cdots \oplus W_{N}$ the corresponding canonical decomposition of $\mathcal{F}^{k}(\mathcal{C})$ into isotypical components. The projection $p_{j}$ of $\mathcal{F}^{k}(\mathcal{C})$ onto $W_{j}$ associated with this decomposition is given by the formula $[\underline{S}$

$$
p_{j}=\frac{d_{j}}{|G|} \sum_{g \in G}\left(\chi_{j}(g)\right)^{\dagger} R_{g}
$$

where $z^{\dagger}$ denotes the complex conjugate of $z \in \mathbb{C}$. Let $\mathcal{A}^{k}(\mathcal{C})=V_{1} \oplus \cdots \oplus V_{N}$ be the decomposition dual to $\mathcal{F}^{k}(\mathcal{C})=W_{1} \oplus \cdots \oplus W_{N}$.

For $j=1, \ldots, N$, define the rational map $v_{j}: \mathbb{C}^{k} \rightarrow W_{j}$, regular on $U$, as the composition of $v$ and $p_{j}$.

Let $\left\{x_{m}\right\}$ be a basis in $W_{j}$ and $\left\{x_{m}^{*}\right\}$ the dual basis in $V_{j}$. If $x_{m}^{*}=u_{m} d t_{1} \wedge \cdots \wedge d t_{k}$, then $v_{j}(t)=\sum_{m} u_{m}(t) x_{m}$. Clearly $v_{1}(t)+\cdots+v_{N}(t)=v(t)$ for $t \in U$.

The map $v_{j}$ will be called the specialization map associated with the isotypical component $W_{j} \subset \mathcal{F}^{k}(\mathcal{C})$.

\section{Corollary 3.6.}

(i) Let $t^{1}, t^{2} \in U$ be isolated critical points of $\Phi$ whose $G$-orbits do not intersect. Then $S^{(a)}\left(v_{j}\left(t^{1}\right), v_{j}\left(t^{2}\right)\right)=0$.

(ii) Let $t \in U$ be an isolated critical point of $\Phi$. Assume that the $G$-orbit of $t$ consists of $|G|$ elements. Then for $j=1, \ldots, N$, we have

$$
S^{(a)}\left(v_{j}(t), v_{j}(t)\right)=c_{j}(-1)^{k} \operatorname{Hess}^{(\mathrm{a})}(t), \quad c_{j}=\frac{\left(d_{j}\right)^{2}}{|G|^{2}} \sum_{g \in G}\left(\left(\chi_{j}(g)\right)^{\dagger}\right)^{2} .
$$

In particular, if $\chi_{j}: G \rightarrow \mathbb{C}$ takes values in $\mathbb{R}$ only, then $c_{j}=\left(d_{j}\right)^{2} /|G|$.

\section{Proof of Theorem 3.1}

4.1. Proof of parts (i) and (ii) of Theorem 3.1. A point $t \in U$ is a critical point of $\Phi$ if and only if the differential 1-form

$$
\omega^{(a)}=\sum_{j \in J(\mathcal{C})} a\left(H_{j}\right) \frac{d f_{j}}{f_{j}}
$$


equals zero at $t$. The form is zero at $t$ if and only if $\langle v(t), \eta\rangle=0$ for all $\eta$ lying in the image of $d_{\mathcal{A}}^{(a)}$, thus if and only if the vector $v(t)$ is singular.

Let $t^{1}, t^{2} \in U$. By definition of the Shapovalov form, we have

$$
S^{(a)}\left(v\left(t^{1}\right), v\left(t^{2}\right)\right)=\sum_{\left\{j_{1}, \ldots, j_{k}\right\} \subset J(\mathcal{C})} D\left(j_{1}, \ldots, j_{k}\right)^{2} \prod_{l=1}^{k} \frac{a\left(H_{j_{l}}\right)}{f_{j_{l}}\left(t^{1}\right) f_{j_{l}}\left(t^{2}\right)},
$$

where $D\left(j_{1}, \ldots, j_{k}\right)=\operatorname{det}_{1 \leq i, l \leq k}\left(b_{j_{l}}^{i}\right)$ and the sum is over all unordered $k$-element subsets in $J(\mathcal{C})$. The right hand side of this formula for $t^{1}=t^{2}=t$ gives $(-1)^{k} \operatorname{det}\left(\frac{\partial^{2}}{\partial t_{i} \partial t_{j}} \ln \Phi\right)(t)$.

4.2. Generic arrangements. An arrangement $\mathcal{C}$ is generic if for any distinct $i_{1}, \ldots, i_{k} \in$ $J(\mathcal{C})$, the intersection $H_{i_{1}} \cap \cdots \cap H_{i_{k}}$ is a point, and for any distinct $i_{1}, \ldots, i_{k+1} \in J(\mathcal{C})$, the intersection $H_{i_{1}} \cap \cdots \cap H_{i_{k+1}}$ is empty.

Fix an ordering on $J(\mathcal{C})$.

For a generic arrangement, a basis in $\mathcal{F}^{k}(\mathcal{C})$ is formed by the flags $F\left(H_{i_{1}}, \ldots, H_{i_{k}}\right)$, $i_{1}, \ldots, i_{k} \in J(\mathcal{C})$, such that $i_{1}<\cdots<i_{k}$. This basis will be called standard.

In $\mathcal{F}^{k}(\mathcal{C})$ we have

$$
F\left(H_{i_{1}}, \ldots, H_{i_{k}}\right)=(-1)^{|\sigma|} F\left(H_{i_{\sigma(1)}}, \ldots, H_{i_{\sigma(k)}}\right)
$$

for any $\sigma \in S_{k}$.

We have

$$
S^{(a)}\left(F\left(H_{i_{1}}, \ldots, H_{i_{k}}\right), F\left(H_{i_{1}}, \ldots, H_{i_{k}}\right)\right)=a\left(H_{i_{1}}\right) \cdots a\left(H_{i_{k}}\right)
$$

and

$$
S^{(a)}\left(F\left(H_{i_{1}}, \ldots, H_{i_{k}}\right), F\left(H_{j_{1}}, \ldots, H_{j_{k}}\right)\right)=0
$$

for distinct elements of the standard basis.

For any distinct $j_{1}, \ldots, j_{k+1} \in J(\mathcal{C}), j_{1}<\cdots<j_{k+1}$, define a linear map $L_{j_{1}, \ldots, j_{k+1}}$ : $\mathcal{F}^{k}(\mathcal{C}) \rightarrow \mathcal{F}^{k}(\mathcal{C})$ by its action on the elements of the standard basis: if $i_{1}, \ldots, i_{k}$ is not a subset of $j_{1}, \ldots, j_{k+1}$, then $F\left(H_{i_{1}}, \ldots, H_{i_{k}}\right) \mapsto 0$, and

$$
F\left(H_{j_{1}}, \ldots, \widehat{H_{j_{p}}}, \ldots, H_{j_{k+1}}\right) \mapsto(-1)^{p} \sum_{l=1}^{k+1}(-1)^{l} a\left(H_{j_{l}}\right) F\left(H_{j_{1}}, \ldots, \widehat{H_{j_{l}}}, \ldots, H_{j_{k+1}}\right) .
$$

Lemma 4.1. The map $L_{j_{1}, \ldots, j_{k+1}}$ is self-adjoint,

$$
S^{(a)}\left(L_{j_{1}, \ldots, j_{k+1}} F_{1}, F_{2}\right)=S^{(a)}\left(F_{1}, L_{j_{1}, \ldots, j_{k+1}} F_{2}\right)
$$

for any $F_{1}, F_{2} \in \mathcal{F}^{k}(\mathcal{C})$.

Fix affine coordinates $t_{i}, i=1, \ldots, k$, on $\mathbb{C}^{k}$ and for $j \in J(\mathcal{C})$ a polynomial

$$
f_{j}\left(t_{1}, \ldots, t_{k}\right)=b_{j}^{0}+b_{j}^{1} t_{1}+\cdots+b_{j}^{k} t_{k}
$$

whose kernel is $H_{j}$. 
Consider $f_{j}, j \in J(\mathcal{C})$, as polynomials in variables $t_{1}, \ldots, t_{k}, b_{j}^{0}, j \in J(\mathcal{C})$. For $j_{1}, \ldots, j_{k+1} \in J(\mathcal{C}), j_{1}<\cdots<j_{k+1}$, introduce polynomials

$$
f_{j_{1}, \ldots, j_{k+1}}=\sum_{p=1}^{k+1} D\left(j_{1}, \ldots, \widehat{j}_{p}, \ldots, j_{k+1}\right) b_{j_{p}}^{0} .
$$

The polynomials $f_{j_{1}}, \ldots, f_{j_{k+1}}, f_{j_{1}, \ldots, j_{k+1}}$ are linearly dependent. Denote $\omega_{j_{1}, \ldots, j_{k+1}}=$ $d f_{j_{1}, \ldots, j_{k+1}} / f_{j_{1}, \ldots, j_{k+1}}$. Then

$$
\omega_{j_{1}} \wedge \cdots \wedge \omega_{j_{k+1}}=\omega_{j_{1}, \ldots, j_{k+1}} \wedge \sum_{p=1}^{k+1}(-1)^{p-1} \omega_{j_{1}} \wedge \cdots \wedge \widehat{\omega_{j_{p}}} \wedge \cdots \wedge \omega_{j_{k+1}}
$$

\section{Lemma 4.2.}

$$
\begin{aligned}
& \sum_{j_{1}<\cdots<j_{k}}\left(\sum_{j} a\left(H_{j}\right) \omega_{j}\right) \wedge \omega_{j_{1}} \wedge \cdots \wedge \omega_{j_{k}} \otimes F\left(H_{j_{1}}, \ldots, H_{j_{k}}\right)= \\
& \sum_{j_{1}<\cdots<j_{k}} \sum_{i_{1}<\cdots<i_{k+1}} \omega_{i_{1}, \ldots, i_{k+1}} \wedge \omega_{j_{1}} \wedge \cdots \wedge \omega_{j_{k}} \otimes L_{i_{1}, \ldots, i_{k+1}} F\left(H_{j_{1}}, \ldots, H_{j_{k}}\right) .
\end{aligned}
$$

For $j \in J(\mathcal{C})$, define a linear map $K_{j}: \mathcal{F}^{k}(\mathcal{C}) \rightarrow \mathcal{F}^{k}(\mathcal{C})$ by the formula

$$
K_{j}=\sum(-1)^{p} \frac{D\left(i_{1}, \ldots, \hat{i_{p}}, \ldots, i_{k+1}\right)}{f_{i_{1}, \ldots, i_{k+1}}} L_{i_{1}, \ldots, i_{k+1}}
$$

where the sum is over all $i_{1}, \ldots, i_{k+1} \in J(\mathcal{C}), i_{1}<\cdots<i_{k+1}$, and $1 \leq p \leq k+1$ such that $i_{p}=j$. The operator $K_{j}$ is self-adjoint.

Lemma 4.3. If $t \in U$ is a critical point of $\Phi$, then for any $j \in J(\mathcal{C})$, the special singular vector $v(t)$ is an eigenvector of $K_{j}$ with eigenvalue $a\left(H_{j}\right) /\left.f_{j}\right|_{t}$.

The lemma follows from Lemma 4.2

Corollary 4.4. If $t^{1}, t^{2} \in U$ are distinct critical points of $\Phi$, then $v\left(t^{1}\right)$ and $v\left(t^{2}\right)$ are orthogonal with respect to $S^{(a)}$.

Now part (iii) of Theorem 3.1 follows from Corollary 4.4 and the continuity of $S^{(a)}\left(v\left(t^{1}\right), v\left(t^{2}\right)\right)$ with respect to deformations of $t^{1}, t^{2}$ and of the arrangement $\mathcal{C}$.

\section{Applications to the Bethe ansatz of the Gaudin model}

5.1. The Gaudin model. Let $\mathfrak{g}$ be a simple Lie algebra over $\mathbb{C}$ with Cartan matrix $A=\left(a_{i, j}\right)_{i, j=1}^{r}$. Let $\mathfrak{h} \subset \mathfrak{g}$ be the Cartan sub-algebra. Fix simple roots $\alpha_{1}, \ldots, \alpha_{r}$ in $\mathfrak{h}^{*}$ and an invariant bilinear form $($,$) on \mathfrak{g}$. Let $H_{1}, \ldots, H_{r} \in \mathfrak{h}$ be the corresponding coroots, $\left\langle\lambda, H_{i}\right\rangle=2\left(\lambda, \alpha_{i}\right) /\left(\alpha_{i}, \alpha_{i}\right)$ for $\lambda \in \mathfrak{h}^{*}$. In particular, $\left\langle\alpha_{j}, H_{i}\right\rangle=a_{i, j}$. 
Let $E_{1}, \ldots, E_{r} \in \mathfrak{n}_{+}, H_{1}, \ldots, H_{r} \in \mathfrak{h}, F_{1}, \ldots, F_{r} \in \mathfrak{n}_{-}$be the Chevalley generators of $\mathfrak{g}$,

$$
\begin{aligned}
{\left[E_{i}, F_{j}\right] } & =\delta_{i, j} H_{i}, \quad i, j=1, \ldots r, \\
{\left[h, h^{\prime}\right] } & =0, \quad h, h^{\prime} \in \mathfrak{h}, \\
{\left[h, E_{i}\right] } & =\left\langle\alpha_{i}, h\right\rangle E_{i}, \quad h \in \mathfrak{h}, i=1, \ldots r, \\
{\left[h, F_{i}\right] } & =-\left\langle\alpha_{i}, h\right\rangle F_{i}, \quad h \in \mathfrak{h}, i=1, \ldots r,
\end{aligned}
$$

and $\left(\operatorname{ad} E_{i}\right)^{1-a_{i, j}} E_{j}=0,\left(\operatorname{ad} F_{i}\right)^{1-a_{i, j}} F_{j}=0$, for all $i \neq j$.

Let $\left(x_{i}\right)_{i \in I}$ be an orthonormal basis in $\mathfrak{g}, \quad \Omega=\sum_{i \in I} x_{i} \otimes x_{i} \in \mathfrak{g} \otimes \mathfrak{g}$ the Casimir element.

For a $\mathfrak{g}$-module $V$ and $\mu \in \mathfrak{h}^{*}$ denote by $V[\mu]$ the weight subspace of $V$ of weight $\mu$ and by $\operatorname{Sing} V[\mu]$ the subspace of singular vectors of weight $\mu$,

$$
\text { Sing } V[\mu]=\left\{v \in V \mid \mathfrak{n}_{+} v=0, h v=\langle\mu, h\rangle v\right\} .
$$

Let $n$ be a positive integer and $\boldsymbol{\Lambda}=\left(\Lambda_{1}, \ldots, \Lambda_{n}\right), \Lambda_{i} \in \mathfrak{h}^{*}$, a set of weights. For $\mu \in \mathfrak{h}^{*}$ let $V_{\mu}$ be the irreducible $\mathfrak{g}$-module with highest weight $\mu$. Denote by $V_{\boldsymbol{\Lambda}}$ the tensor product $V_{\Lambda_{1}} \otimes \cdots \otimes V_{\Lambda_{n}}$.

If $X \in$ End $\left(V_{\Lambda_{i}}\right)$, then we denote by $X^{(i)} \in$ End $\left(V_{\boldsymbol{\Lambda}}\right)$ the operator $\cdots \otimes \mathrm{id} \otimes X \otimes \mathrm{id} \otimes \cdots$ acting non-trivially on the $i$-th factor of the tensor product only. If $X=\sum_{m} X_{m} \otimes Y_{m} \in$ End $\left(V_{\Lambda_{i}} \otimes V_{\Lambda_{j}}\right)$, then we set $X^{(i, j)}=\sum_{m} X_{m}^{(i)} \otimes Y_{m}^{(j)} \in \operatorname{End}\left(V_{\Lambda}\right)$.

Let $z=\left(z_{1}, \ldots, z_{n}\right)$ be a point in $\mathbb{C}^{n}$ with distinct coordinates. Introduce linear operators $K_{1}(z), \ldots, K_{n}(z)$ on $V_{\boldsymbol{\Lambda}}$ by the formula

$$
K_{i}(z)=\sum_{j \neq i} \frac{\Omega^{(i, j)}}{z_{i}-z_{j}}, \quad i=1, \ldots, n .
$$

The operators are called the Gaudin Hamiltonians of the Gaudin model associated with $V_{\boldsymbol{\Lambda}}$. The Hamiltonians commute, $\left[K_{i}(z), K_{j}(z)\right]=0$ for all $i, j$.

The main problem for the Gaudin model is to diagonalize simultaneously the Hamiltonians.

One can check that the Hamiltonians commute with the action of $\mathfrak{g}$ on $V_{\boldsymbol{\Lambda}}$. Therefore it is enough to diagonalize the Hamiltonians on the subspaces of singular vectors Sing $V_{\boldsymbol{\Lambda}}[\mu] \subset V_{\boldsymbol{\Lambda}}$.

The eigenvectors of the Gaudin Hamiltonians are constructed by the Bethe ansatz method. We remind the construction in the next section.

5.2. Master functions and the canonical weight function, c.f. [MV4. Fix a collection of weights $\boldsymbol{\Lambda}=\left(\Lambda_{1}, \ldots, \Lambda_{n}\right), \Lambda_{i} \in \mathfrak{h}{ }^{*}$, and a collection of non-negative integers $\boldsymbol{k}=\left(k_{1}, \ldots, k_{r}\right)$. Denote $k=k_{1}+\cdots+k_{r}, \Lambda=\Lambda_{1}+\cdots+\Lambda_{n}$, and $\alpha(\boldsymbol{k})=k_{1} \alpha_{1}+\cdots+k_{r} \alpha_{r}$.

Let $c$ be the unique non-decreasing function from $\{1, \ldots, k\}$ to $\{1, \ldots, r\}$, such that $\# c^{-1}(i)=k_{i}$ for $i=1, \ldots, r$. The master function $\Phi(t, z, \boldsymbol{\Lambda}, \boldsymbol{l})$ associated with this data 
is defined by the formula

$$
\Phi(t, z, \boldsymbol{\Lambda}, \boldsymbol{k})=\prod_{1 \leq i<j \leq n}\left(z_{i}-z_{j}\right)^{\left(\Lambda_{i}, \Lambda_{j}\right)} \prod_{i=1}^{l} \prod_{s=k}^{n}\left(t_{i}-z_{s}\right)^{-\left(\alpha_{c(i)}, \Lambda_{s}\right)} \prod_{1 \leq i<j \leq k}\left(t_{i}-t_{j}\right)^{\left(\alpha_{c(i)}, \alpha_{c(j)}\right)},
$$

see $[\underline{S V}]$. The function $\Phi$ is a function of complex variables $t=\left(t_{1}, \ldots, t_{k}\right), z=$ $\left(z_{1}, \ldots, z_{n}\right)$, weights $\boldsymbol{\Lambda}$, and discrete parameters $\boldsymbol{k}$. The main variables are $t$, the other variables will be considered as parameters.

For given $z, \boldsymbol{\Lambda}, \boldsymbol{k}$, a point $t \in \mathbb{C}^{k}$ is a critical point of the master function if the following system of algebraic equations is satisfied,

$$
-\sum_{s=1}^{n} \frac{\left(\alpha_{c(i)}, \Lambda_{s}\right)}{t_{i}-z_{s}}+\sum_{j, j \neq i} \frac{\left(\alpha_{c(i)}, \alpha_{c(j)}\right)}{t_{i}-t_{j}}=0, \quad i=1, \ldots, k .
$$

Let $\Sigma_{k}$ be the permutation group of the set $\{1, \ldots, k\}$. Denote by $\Sigma_{\boldsymbol{k}} \subset \Sigma_{k}$ the subgroup of all permutations preserving the level sets of the function $c$. The subgroup $\boldsymbol{\Sigma}_{\boldsymbol{k}}$ is isomorphic to $\Sigma_{k_{1}} \times \cdots \times \Sigma_{k_{r}}$ and acts on $\mathbb{C}^{k}$ permuting coordinates of $t$. The action of the subgroup $\Sigma_{\boldsymbol{k}}$ preserves the critical set of the master function. All orbits of the action of $\boldsymbol{\Sigma}_{\boldsymbol{k}}$ on the critical set have the same cardinality $k_{1} ! \cdots k_{r}$ ! .

Consider highest weight irreducible $\mathfrak{g}$-modules $V_{\Lambda_{1}}, \ldots, V_{\Lambda_{n}}$, the tensor product $V_{\boldsymbol{\Lambda}}=$ $V_{\Lambda_{1}} \otimes \cdots \otimes V_{\Lambda_{n}}$, and its weight subspace $V_{\boldsymbol{\Lambda}}[\Lambda-\alpha(\boldsymbol{k})]$. Fix a highest weight vector $v_{\Lambda_{i}}$ in $V_{\Lambda_{i}}$ for all $i$.

We construct a rational map

$$
\omega: \mathbb{C}^{k} \times \mathbb{C}^{n} \rightarrow V_{\Lambda}[\Lambda-\alpha(\boldsymbol{k})]
$$

called the canonical weight function.

Let $P(\boldsymbol{k}, n)$ be the set of sequences $I=\left(i_{1}^{1}, \ldots, i_{j_{1}}^{1} ; \ldots ; i_{1}^{n}, \ldots, i_{j_{n}}^{n}\right)$ of integers in $\{1, \ldots, r\}$ such that for all $i=1, \ldots, r$, the integer $i$ appears in $I$ precisely $k_{i}$ times. For $I \in P(\boldsymbol{k}, n)$, and a permutation $\sigma \in \Sigma_{k}$, set $\sigma_{1}(i)=\sigma(i)$ for $i=1, \ldots, j_{1}$, and $\sigma_{s}(i)=\sigma\left(j_{1}+\cdots+j_{s-1}+i\right)$ for $s=2, \ldots, n$ and $i=1, \ldots, j_{s}$.

Define

$$
\Sigma(I)=\left\{\sigma \in \Sigma_{k} \mid c\left(\sigma_{s}(j)\right)=i_{s}^{j} \text { for } s=1, \ldots, n \text { and } j=1, \ldots j_{s}\right\} .
$$

To every $I \in P(\boldsymbol{k}, n)$ we associate a vector

$$
F_{I} v=F_{i_{1}^{1}} \ldots F_{i_{j_{1}}^{1}} v_{\Lambda_{1}} \otimes \cdots \otimes F_{i_{1}^{n}} \ldots F_{i_{j_{n}}^{n}} v_{\Lambda_{n}}
$$

in $V_{\boldsymbol{\Lambda}}[\Lambda-\alpha(\boldsymbol{k})]$, and rational functions

$$
\omega_{I, \sigma}=\omega_{\sigma_{1}(1), \ldots, \sigma_{1}\left(j_{1}\right)}\left(z_{1}\right) \cdots \omega_{\sigma_{n}(1), \ldots, \sigma_{n}\left(j_{n}\right)}\left(z_{n}\right),
$$

labeled by $\sigma \in \Sigma(I)$, where

$$
\omega_{i_{1}, \ldots, i_{j}}\left(z_{s}\right)=\frac{1}{\left(t_{i_{1}}-t_{i_{2}}\right) \cdots\left(t_{i_{j-1}}-t_{i_{j}}\right)\left(t_{i_{j}}-z_{s}\right)} .
$$


We set

$$
\omega(z, t)=\sum_{I \in P(\boldsymbol{k}, n)} \sum_{\sigma \in \Sigma(I)} \omega_{I, \sigma} F_{I} v .
$$

The canonical weight function was introduced in [SV] to solve the KZ equations, see [SV, FSV2, FMTV]. The hypergeometric solutions to the KZ equations with values in Sing $V_{\boldsymbol{\Lambda}}[\Lambda-\alpha(\boldsymbol{k})]$ have the form

$$
I(z)=\int_{\gamma(z)} \Phi(t, z, \boldsymbol{\Lambda}, \boldsymbol{k})^{1 / \kappa} \omega(t, z) d t .
$$

Different formulas for the canonical weight function see in [RSV].

The values of the canonical weight function at the critical points (with respect to variables $t$ ) of the master function are called the Bethe vectors, see [RV, V2, FFR.

Theorem $5.1([\mathrm{RV}])$. Assume that $z \in \mathbb{C}^{n}$ has distinct coordinates. Assume that $t \in \mathbb{C}^{k}$ is a critical point of the master function $\Phi(., z, \boldsymbol{\Lambda}, \boldsymbol{k})$. Then the vector $\omega(t, z)$ belongs to Sing $V_{\boldsymbol{\Lambda}}[\Lambda-\alpha(\boldsymbol{k})]$ and is an eigenvector of the Gaudin Hamiltonians $K_{1}(z), \ldots, K_{n}(z)$.

This theorem was proved in [RV] using the quasi-classical asymptotics of the hypergeometric solutions of the KZ equations. The theorem also follows directly from Theorem 6.16.2 in [SV], cf. Theorem 7.2.5 in [SV], see also Theorem 4.2.2 in [FSV2].

5.3. The Shapovalov Form. Define the anti-involution $\tau: \mathfrak{g} \rightarrow \mathfrak{g}$ sending $E_{1}, \ldots, E_{r}$, $H_{1}, \ldots, H_{r}, F_{1}, \ldots, F_{r}$ to $F_{1}, \ldots, F_{r}, H_{1}, \ldots, H_{r}, E_{1}, \ldots, E_{r}$, respectively.

Let $W$ be a highest weight $\mathfrak{g}$-module with highest weight vector $w$. The Shapovalov form on $W$ is the unique symmetric bilinear form $S$ defined by the conditions:

$$
S(w, w)=1, \quad S(x u, v)=S(u, \tau(x) v)
$$

for all $u, v \in W$ and $x \in \mathfrak{g}$, see $[\mathrm{K}$.

Let $V_{\Lambda_{1}}, \ldots, V_{\Lambda_{n}}$ be irreducible highest weight modules and $V_{\boldsymbol{\Lambda}}$ their tensor product. Let $v_{\Lambda_{i}} \in V_{\Lambda_{i}}$ be a highest weight vector and $S_{i}$ the corresponding Shapovalov form on $V_{\Lambda_{i}}$. Define a symmetric bilinear form on $V_{\boldsymbol{\Lambda}}$ by the formula

$$
S=S_{1} \otimes \cdots \otimes S_{n} .
$$

The form $S$ will be called the tensor product Shapovalov form on $V_{\boldsymbol{\Lambda}}$.

5.4. Application of Theorem [3.1. As in Section 5.2 fix a collection of weights $\boldsymbol{\Lambda}=$ $\left(\Lambda_{1}, \ldots, \Lambda_{n}\right), \Lambda_{i} \in \mathfrak{h}^{*}$, and a collection of non-negative integers $\boldsymbol{k}=\left(k_{1}, \ldots, k_{r}\right)$.

Let $S_{V_{\boldsymbol{\Lambda}}}$ be the tensor product Shapovalov from on the tensor product $V_{\boldsymbol{\Lambda}}$.

Fix a collection of distinct complex numbers $z=\left(z_{1}, \ldots, z_{n}\right)$.

Let $t^{1}, t^{2} \in \mathbb{C}^{k}$ be points such that $t^{1}$ has distinct coordinates and $t^{2}$ has distinct coordinates and such that none of coordinates of $t^{1}, t^{2}$ belongs to the set $\left\{z_{1}, \ldots, z_{n}\right\}$.

Under these assumptions, we have the following theorem.

\section{Theorem 5.2.}


(i) Assume that $t^{1}$ and $t^{2}$ are isolated critical points of $\Phi(., z, \boldsymbol{\Lambda}, \boldsymbol{k})$. Assume that the $\boldsymbol{\Sigma}_{\boldsymbol{k}}$-orbits of $t^{1}$ and $t^{2}$ do not intersect. Then the Bethe vectors $\omega\left(z, t^{1}\right)$ and $\omega\left(z, t^{2}\right)$ are orthogonal with respect to the tensor product Shapovalov form, $S_{V_{\Lambda}}\left(\omega\left(z, t^{1}\right), \omega\left(z, t^{2}\right)\right)=0$.

(ii) Assume that $t^{1}$ is an isolated critical point of $\Phi(., z, \boldsymbol{\Lambda}, \boldsymbol{k})$. Then

$$
S_{V_{\boldsymbol{\Lambda}}}\left(\omega\left(z, t^{1}\right), \omega\left(z, t^{1}\right)\right)=\operatorname{det}_{1 \leq i, j \leq k}\left(\frac{\partial^{2}}{\partial t_{i} \partial t_{j}} \ln \Phi\left(t^{1}, z, \boldsymbol{\Lambda}, \boldsymbol{k}\right)\right) .
$$

(iii) Let d be a natural number. Let $C$ be a set of d distinct $\boldsymbol{\Sigma}_{\boldsymbol{k}}$-orbits of nondegenerate critical points of $\Phi$. Choose a representative $t^{i}$ in each orbit. Assume that each point $t^{i}$ has distinct coordinates and none of the coordinates of $t^{i}$ belongs to the set $\left\{z_{1}, \ldots, z_{n}\right\}$. Then the Bethe vectors $\omega\left(z, t^{i}\right), i=1, \ldots \mathrm{d}$, span $a$ d-dimensional subspace in $\operatorname{Sing} V_{\boldsymbol{\Lambda}}[\Lambda-\alpha(\boldsymbol{k})]$.

Part (i) of the theorem was proved in $\left[\mathrm{RV}\right.$ for $\mathfrak{g}=s l_{2}$. Part (ii) was proved for $\mathfrak{g}=s l_{r+1}, r=1$, in [V2 and for arbitrary $r$ in [MV4]. In all those cases the proof used asymptotics of Bethe vectors in the asymptotic zone $\left|z_{1}-z_{2}\right|<<\cdots<<\left|z_{1}-z_{n}\right|$.

Part (iii) gives a bound from above on the number $d$ of orbits of non-degenerate critical points of $\Phi(., z, \boldsymbol{\Lambda}, \boldsymbol{k})$ in terms of the representation theory. In particular, if the weight $\Lambda-\alpha(\boldsymbol{k})$ is not integral dominant, then $\Phi(., z, \boldsymbol{\Lambda}, \boldsymbol{k})$ does not have at all non-degenerate critical points (since in that case the space Sing $V_{\boldsymbol{\Lambda}}[\Lambda-\alpha(\boldsymbol{k})]$ has dimension zero).

It is interesting to note that if $\Lambda-\alpha(\boldsymbol{k})$ is not integral dominant, then all critical points of $\Phi(., z, \boldsymbol{\Lambda}, \boldsymbol{k})$ are non-isolated and the connected components of the critical set are isomorphic to suitable Bruhat cells of the flag variety of the Langlands dual Lie algebra, see ScV, MV2, MV3].

In $[\mathrm{ScV}]$ the case of $\mathfrak{g}=s l_{2}$ was considered. It was proved that if the weight $\Lambda-\alpha(\boldsymbol{k})$ is integral dominant and $z_{1}, \ldots, z_{n}$ are generic, then the function $\Phi(., z, \boldsymbol{\Lambda}, \boldsymbol{k})$ has nondegenerate critical points only and the critical points form $d$ orbits, where $d$ is the dimension of Sing $V_{\boldsymbol{\Lambda}}[\Lambda-\alpha(\boldsymbol{k})]$. In particular, this means that the corresponding Bethe vectors form a basis in Sing $V_{\boldsymbol{\Lambda}}[\Lambda-\alpha(\boldsymbol{k})]$.

5.5. Proof of Theorem [5.2. For given $z=\left(z_{1}, \ldots, z_{n}\right)$, the discriminantal arrangement $\mathcal{C}(z)$ in $\mathbb{C}^{k}$ is defined as the collection of hyperplanes

$H_{i}^{s}: t_{i}-z_{s}=0(i=1, \ldots, k, s=1, \ldots, n), \quad H_{i, j}: t_{i}-t_{j}=0(1 \leq i<j \leq k)$,

see [SV]. Define the weights of $\mathcal{C}(z)$ by the rule, $a\left(H_{i}^{s}\right)=-\left(\alpha_{i}, \Lambda_{s}\right), a\left(H_{i, j}\right)=-\left(\alpha_{i}, \alpha_{j}\right)$. Then the master function $\Phi(., z, \boldsymbol{\Lambda}, \boldsymbol{k})$ is equal up to a constant factor to the master function $\Phi$, defined in Section 3.1 and assigned to the weighted arrangement $\mathcal{C}(z)$.

Let $S^{(a)}: \mathcal{F}^{k}(\mathcal{C}(z)) \otimes \mathcal{F}^{k}(\mathcal{C}(z)) \rightarrow \mathbb{C}$ be the Shapovalov form of $\mathcal{C}(z)$.

The group $\Sigma_{\boldsymbol{k}}$ acts on $\mathbb{C}^{k}$ permuting coordinates. The action preserves the discriminantal arrangement and its weights. Hence the group acts on $\mathcal{F}^{k}(\mathcal{C}(z))$ and $\mathcal{A}^{k}(\mathcal{C}(z))$. Set

$$
W^{-}=\left\{x \in \mathcal{F}^{k}(\mathcal{C}(z)) \mid R_{\sigma}(x)=(-1)^{|\sigma|} x, \sigma \in \boldsymbol{\Sigma}_{\boldsymbol{k}}\right\}
$$


Similarly define $V^{-} \subset \mathcal{A}^{k}(\mathcal{C}(z))$ to be the skew-symmetric part of $\mathcal{A}^{k}(\mathcal{C}(z))$. The subspaces $V^{-}$and $W^{-}$are dual.

For an element $I=\left(i_{1}^{1}, \ldots, i_{j_{1}}^{1} ; \ldots ; i_{1}^{n}, \ldots, i_{j_{n}}^{n}\right)$ in $P(\boldsymbol{k}, n)$ and a permutation $\sigma$ in $\Sigma(I)$ define a flag $f_{I, \sigma} \in \mathcal{F}^{k}(\mathcal{C}(z))$,

$$
f_{I, \sigma}=F\left(H_{\sigma_{1}(1)}^{1}, \ldots, H_{\sigma_{1}\left(j_{1}\right)}^{1}, \ldots, H_{\sigma_{n}(1)}^{n}, \ldots, H_{\sigma_{1}\left(j_{n}\right)}^{n}\right),
$$

and then an element $f_{I} \in W^{-}$,

$$
f_{I}=\frac{1}{k_{1} ! \ldots k_{r} !} \sum_{\sigma \in \Sigma(I)}(-1)^{|\sigma|} f_{I, \sigma} .
$$

Theorem 5.3 ( Theorem 6.6 in $[\mathrm{SV}]$ ). For $I, J \in P(\boldsymbol{k}, n)$,

$$
S_{V_{\Lambda}}\left(F_{I} v, F_{J} v\right)=(-1)^{k} k_{1} ! \ldots k_{n} ! S^{(a)}\left(f_{I}, f_{J}\right) .
$$

Theorem 5.4. The element

$$
\sum_{I \in P(\boldsymbol{k}, n)}\left(\sum_{\sigma \in \Sigma(I)} \omega_{I, \sigma} d t_{1} \wedge \ldots d t_{k}\right) \otimes F_{I} v
$$

is the canonical element in $V^{-} \otimes W^{-}$.

The theorem is a direct corollary of Theorems 5.13 and 6.16.2 in [SV].

Let $v^{-}: \mathbb{C}^{k} \rightarrow W^{-}$be the specialization map associated with the isotypical component $W^{-} \subset \mathcal{F}^{k}(\mathcal{C}(z))$. Let $t^{1}, t^{2}$ be as in Theorem [5.2, then

$$
S_{V_{\Lambda}}\left(\omega\left(z, t^{1}\right), \omega\left(z, t^{2}\right)\right)=(-1)^{k} k_{1} ! \ldots k_{n} ! S^{(a)}\left(v^{-}\left(t^{1}\right), v^{-}\left(t^{2}\right)\right)
$$

by Theorems 5.3 and 5.4. By Corollary 3.6 the right hand side is zero if the orbits of $t^{1}$ and $t^{2}$ do not intersect. By Corollary [3.6, we have

$$
(-1)^{k} k_{1} ! \ldots k_{n} ! S^{(a)}\left(v^{-}\left(t^{1}\right), v^{-}\left(t^{1}\right)\right)=\operatorname{det}_{1 \leq i, j \leq k}\left(\frac{\partial^{2}}{\partial t_{i} \partial t_{j}} \ln \Phi\left(t^{1}, z, \boldsymbol{\Lambda}, \boldsymbol{k}\right)\right) .
$$

This proves parts (i) and (ii) of Theorem 5.2. Part (iii) follows from the fact that vectors $\omega\left(z, t^{i}\right), i=1, \ldots, d$, have non-zero Shapovalov norm and are pair-wise orthogonal.

\section{REFERENCES}

[A] K. Aomoto, On vanishing of cohomology attached to certain many valued meromorphic functions, J. Math. Soc. Japan 27 (1975), 248-255.

[B] H. Babujian, Off-shell Bethe ansatz equations and $N$-point correlators in the $\mathrm{SU}(2)$ WZNW theory, J. Phys. A 26 (1993), no. 23, 6981-6990.

[BF] H. Babujian and R. Flume, Off-shell Bethe ansatz equation for Gaudin magnets and solutions of Knizhnik-Zamolodchikov equations, Modern Phys. Lett. A 9 (1994), n. 22, 2029-2039.

[Fa] L. D. Faddeev, Lectures on Quantum Inverse Scattering Method in Integrable Systems, ed. by X.-C. Song, Nankai Lectures Math Phys., World Scientific, 1990, 23-70.

[BIK] N.M. Bogoliubov, A.G. Izergin, and V.E. Korepin, Quantum Inverse Scattering Method and Correlation Functions, Cambridge University Press, Cambridge, 1993. 
[FT] L. D. Faddeev and L.A. Takhtajan, Quantum Inverse Problem Method and the Heisenberg XYZmodel, Russian Math. Surveys 34 (1979), 11-68.

[FFR] B. Feigin, E. Frenkel, and N. Reshetikhin, Gaudin model, Bethe Ansatz and Critical Level, Commun. Math. Phys. 166 (1994),29-62.

[FSV1] B. Feigin, V. Schechtman, and A. Varchenko, On algebraic equations satisfied by hypergeometric correlators in WZW models. I, Comm. Math. Phys. 163 (1994), 173-184.

[FSV2] B. Feigin, V. Schechtman, and A. Varchenko, On algebraic equations satisfied by hypergeometric correlators in WZW models. II, Comm. in Math. Phys. 170 (1995), 219-247.

[FMTV] G. Felder, Y. Markov, V. Tarasov, and A. Varchenko, Differential Equations Compatible with KZ Equations, QA/0001184, Journal of Math. Phys., Analysis and Geometry, 3 (2000), 139-177.

[F] E. Frenkel, Affine Algebras, Langlands Duality and Bethe Ansatz, math.QA/9506003 (1999), 1-34.

[G] M. Gaudin, Diagonalisation d'une classe d'Hamiltoniens de spin, J. Physique 37 (1976), no. 10, 1089-1098.

[K] V. Kac, Infinite-dimensional Lie algebras, Cambridge University Press, 1990.

[Ko] V. Korepin, Calculation of Norms of Bethe Wave Functions, Comm. Math. Phys. 86 (1982), 391-418.

[MV1] E. Mukhin and A. Varchenko, Remarks on critical points of phase functions and norms of Bethe vectors, Adv. Studies in Pure Math. 27 (2000), Arrangements - Tokyo 1998, 239-246.

[MV2] E. Mukhin and A. Varchenko, Critical Points of Master Functions and Flag Varieties, math.QA/0209017 (2002), 1-49.

[MV3] E. Mukhin and A. Varchenko, Miura Opers and Critical Points of Master Functions, math.QA/0312 (2003), 1-27.

[MV4] E. Mukhin and A. Varchenko, Norm of a Bethe Vector and the Hessian of the Master Function, math.QA/0402349, 1-23.

[OT] P. Orlik and H. Terao, The number of critical points of a product of powers of linear functions, Invent. Math. 120 (1995), no. 1, 1-14.

[R] N. Reshetikhin, Calculation of Norms of Bethe Vectors in Models with SU(3)-symmetry, Zapiski Nauch. Semin. LOMI, 150 (1986), 196-213.

[RV] N. Reshetikhin and A. Varchenko, Quasiclassical asymptotics of solutions to the KZ equations, Geometry, Topology and Physics for R. Bott, Intern. Press, 1995, 293-322.

[RSV] R. Rimanyi, L. Stevens, and A. Varchenko, Combinatorics of rational functions and PoincareBirchoff-Witt expansions of the canonical $U\left(\mathfrak{n}_{-}\right)$-valued differential form, math.CO/0407101, 1-14.

[S] J.-P. Serre, Linear Representations of Finite Groups, Springer-Verlag, 1977.

[Si] R. Silvotti, On a conjecture of Varchenko, Invent. Math. 126 (1996), no. 2, 235-248.

[STV] V. Schechtman, H. Terao, and A. Varchenko, Local systems over complements of hyperplanes and the Kac-Kazhdan conditions for singular vectors, J. Pure Appl. Algebra 100 (1995), no. 1-3, 93-102.

[SV] V. Schechtman and A. Varchenko, Arrangements of hyperplanes and Lie algebra homology, Invent. Math., 106 (1991), 139-194.

$[\mathrm{ScV}]$ I. Scherbak and A. Varchenko, Critical point of functions, sl ${ }_{2}$ representations and Fuchsian differential equations with only univalued solutions, Moscow Math. J., 3, n. 2 (2003), 621-645.

[TV] V. Tarasov and A. Varchenko, Asymptotic Solutions to the Quantized Knizhnik-Zamolodchikov Equation and Bethe Vectors, Amer. Math. Soc. Transl.(2) Vol. 174, 1996, 235-273.

[V1] A. Varchenko, Multidimensional Hypergeometric Functions and Representation Theory of Lie Algebras and Quantum Groups, Advanced Series in Mathematical Physics, Vol. 21, World Scientific, 1995.

[V2] A. Varchenko, Critical points of the product of powers of linear functions and families of bases of singular vectors, Compos. Math., 97 (1995), 385-401. 
[V3] A. Varchenko, Special functions, KZ type equations, and representation theory, CBMS Regional Conference Series in Mathematics, 98, AMS, 2003. 